\title{
Individual Differences in Virtual Environments
}

\author{
Corina Sas \\ University College Dublin, Belfield, Dublin 4, Ireland
}

\begin{abstract}
This paper summarises the results of several studies of individual differences among users navigating in virtual environments. These differences relate to performance of navigational tasks, and the degree of sense of presence experienced by the users. The individual differences addressed in this paper refer primarily to personality and demographic factors. The possibility of improving the design of virtual environments for a better accommodation of these differences is discussed.
\end{abstract}

\section{Introduction}

Individual differences is an umbrella term describing an entire field of research primarily involving psychology that focuses on aspects of behaviour that differentiate individuals from one another. Any attempt to accommodate individual differences should follow after they have been identified and after we have established the aspects with respect to which the individual differences emerged.

The work presented in this paper focuses on two major aspects which usually describe any interaction with a Virtual Environment (VE), such as navigation, and sense of presence.

Because of its prevalence, there is a tendency to take the navigational process for granted. However, navigation is a complex activity which requires seamless integration of several cognitive processes. The difficulties associated with navigation become especially obvious when it is performed in unfamiliar environments (i.e. one becomes lost). Due to their specific characteristics, VEs put additional demands on untrained users. In addition, within VEs the user set of actions is restricted, consisting mainly of navigation and locomotion, object selection, manipulation, modification and query 10 .

Sense of presence is a psychological adjacent phenomenon experienced by users while (and not only) they interact with virtual reality systems.

This paper summarises the findings of some of our previous studies, based on which a set of factors that led to individual differences with respect to previously mentioned aspects, e.g. performance on spatial tasks and sense of presence, have been identified.

\section{Individual Differences}

Clinical studies involve an in-depth investigation of one or a limited number of subjects in order to highlight their individual unique profile. A more general 
approach to studying individual differences, and the one taken by this paper, strives to capture dimensions of individual differences rather than individual patterns. Accordingly, one can group subjects together, based on the commonly shared variance with respect to a particular aspect of their individuality. Such an approach leads to the identification of differences between groups of subjects (intra-group differences) as opposed to the differences among individuals within the same group (inter-group differences).

Identifying and consequently describing groups of individuals sharing common features is a preliminary stage in this kind of research. It leads to a descriptive taxonomy regarding the factors which trigger the individual differences. Attempts to highlight a causal relationship between these factors and other relevant aspects define another area of potential research in this field. The third area of research in this area is a theoretical one, aiming at explaining the structure and dynamics of individual differences [18].

\section{Individual Differences in Virtual Environments}

A significant amount of work has been carried out on individual differences in the area of Human Computer Interaction (HCI). The theoretical and empirical findings in fields like differential psychology and cognitive psychology present a great potential for the researchers in HCI [7]. Carroll's [2] work involved a hierarchical exploratory analysis of part of the studies in differential psychology. His findings regarding individual differences refer to general intelligence as a top level, which includes eight general abilities. Personality traits and cognitive style have been also considered of potential interest for HCI field.

Most of studies related to spatial navigation addressed the issue of individual differences with respect to navigation in abstract information space, such as hypermedia space or semantic space [43]. However, less research has been carried out in the area of spatial navigation within VE.

The basic goal of Virtual Environments (VEs) is to create a place for people to act [28]. An additional purpose, related primarily with the VEs developed for training spatial skills, addresses the users' ability to both learn and represent the spatial characteristics of such virtual spaces [29]. While considerable amount of work concerned the technological factors regarding the design of VEs, factors which might be related to the training effectiveness, little research has been done in the field of user characteristics. As Waller highlighted 29], this is unfortunate since individual differences are a major source of variation in performance in both real and virtual spatial tasks.

A major aspect related to VEs, which can greatly benefit from the insights into the psychology of individual differences, regards the possible transfer of skills from the VE to the real world. Since the inter-subjects variability in performance within a VE is higher than that for the analogous real tasks, it seems that knowledge acquired within a VE requires not only abilities for similar real tasks, but additional skills as well [29. 
A major distinction among different types of VEs refers to immersive and non-immersive VEs. As opposed to non-immersive VEs, immersive VEs involve the restriction of users' senses in terms of their reference to the real world [8]. A desktop VE has been used in the studies summarised within this paper.

\section{Study Design}

The VE which has been used as an experimental testbed across our studies represents a training environment supporting technicians in the maintenance of engineering artefacts [17. The ECHOES system consists of a virtual multistorey building, each of the seven levels containing three rooms, e.g. lobby room, conference room, training room, library etc. Figures 1 and 2 presents a bird's eye view of the ground floor and first floor respectively.

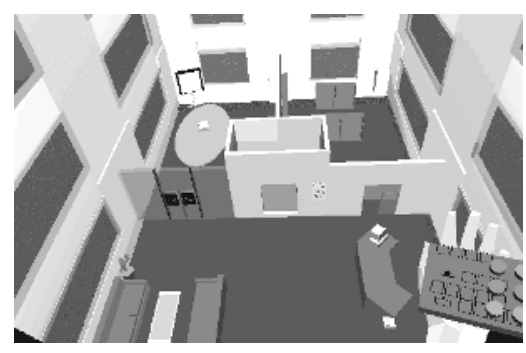

Fig. 1. Bird's Eye View of Ground Floor

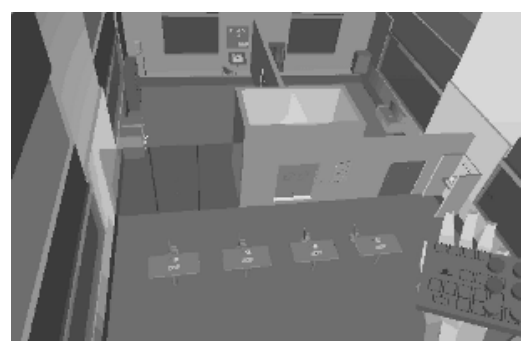

Fig. 2. Bird's Eye View of First Floor

The study involved three phases: familiarisation, exploration and performance measurement. Initially, users were allowed to become accustomed with the VE and to learn movement control. After this, they were asked to perform an exploration task within the virtual building which lasted for approximately 25 minutes. After the completion of this task, during which participants implicitly acquired spatial knowledge related to the VE, they were tested. Given the findings provided by studies on human spatial cognition, the strength of relationship between spatial abilities measured by psychometric tests and spatial knowledge acquisition from VE is arguable [29]. Therefore, in order to assess the level of spatial knowledge acquisition, we employed a search task. Users were placed on the third level and asked to find a particular room identified only through its name, and located on the ground floor.

The time needed to accomplish this task acted as an indicator of the level of spatial knowledge acquired within the VE. According to the time required for the search task, users have been identified as low spatial users, when they needed significantly longer time to find the library (Mean $=49$ seconds), or high spatial users who found the library straight away (Mean $=7$ seconds). 


\section{Individual Differences on Spatial Task Performance}

The demographic factors which we considered of interest in understanding performances on the search task, were gender and prior computer games experience, since all participants were already familiar with computers. Findings indicate significant gender differences: male subjects outperformed female subjects by finding the room significantly quicker. These differences follow the line of findings in the area of spatial abilities that indicate better performances for males [1].

Understanding users' preferred manner of processing information opens a door towards their perception of the world, either physical or virtual. Several studies consider cognitive style and/or learning style important variable(s) potentially triggering individual differences in the way users perform [9]. However, little attention has been paid to the impact of personality cognitive style, defined in terms of Jung's personality types 13 . We conjectured that is might be a significant factor which could lead to differences on spatial task performance.

Without being statistically significant, findings suggest that individuals who are more extrovert, intuitive or thinking perform better in terms of task efficiency. Along the Extroversion-Introversion continuum, the extravert people are predominantly orientated towards the external world, thing which probably leads to an increased level of spatial awareness. On the Sensing-Intuition continuum, intuitive people look at the entire picture which emerges from the single parts, but also go beyond it. The search task is a complex one and its solution requires information perceived through our senses. However, this does not suffice and user has to perceive something which is beyond the immediate information. In order to be successful, one needs an internal representation of the spatial layout, or a so-called cognitive map. Most likely intuitive individuals are able to take the bird's eye view, as they would see the space from above. However, further work is required for validating this hypothesis. Along the Thinking-Feeling continuum, thinking type grounds his/her decision on logic and analysis. The need of thinking type to organise both things and ideas within its environment could prove beneficial in understanding it. Probably when both extrovert and thinking types are met within the same individual, he/she could achieve higher performance in building up the internal representation of the spatial layout through methodical coverage of the space.

\section{Individual Differences on Navigational Pattern}

Ford 9] investigated the individual differences in behavioural patterns of navigating in abstract VEs, triggered by cognitive style defined in terms of holist or serialist orientation. He showed that while holists like to have access to an overview of the underlying structure, serialists prefer keyword indices. As the best of our knowledge there have been no research on identifying patterns of human navigational behaviour within VEs.

Work carried out in one of our studies involved the use of self-organising maps for classifying users' trajectories [22. This allowed identifying a set of 
five clusters, based on the shape of motion trajectory. These clusters helped differentiating study participants on the basis of efficiency of their navigational strategies. Clustering allowed the identification of good and poor motion trajectories and their associated characteristics, where good and poor have been determined in the light of users' performance.

Each cluster but one could prove beneficial for the performance of the search task, excepting one, which consisted of erratic trajectories, presenting lots of turns and straight line segments joined at sharp angles. Since more than 50\% of trajectories composing this cluster belong to the subjects with the worst performance on the search task, we considered trajectories within this cluster as poor trajectories.

These findings indicate that there are individual differences in navigational patterns, which correlate with performance on spatial task, or in other words with users' spatial abilities. While high spatial users perform a good coverage of the space, by following a smooth path which resembles "going around the edge" feature, low spatial users navigate more erratically, performing more greater turns or crossovers. They seem to explore the environment in an unsystematic manner, which is reflected on navigation behaviour both on each level and across the levels. An analysis of these findings in the light of Ford's previously summarised results 9] determined us to conjecture that, through their pattern of navigation, high spatial users developed holistic strategies of exploration, while low spatial users seemed to be more serialistic in their approach.

\section{Individual Differences on Experiencing Presence}

One of the psychological phenomena experienced by users while they interact with virtual reality systems is sense of presence. It allows them to be there [23], to feel themselves immersed and moreover to perceive the virtual world as another world where they really exist. We defined presence as a psychological phenomenon, through which one's cognitive processes are oriented towards another world, either technologically mediated or imaginary, to such an extent that he or she experiences mentally the state of being (there), similar to one in the physical reality, together with an imperceptible shifting of focus of consciousness to the proximal stimulus located in that other world [19. For measuring presence, we devised a questionnaire presented in Sas and O'Hare [21].

Within immersive VEs, most of the users become immersed, despite the intragroup variability based on cognitive factors. We conjecture that in this case, the presence is experienced because of the advanced technological aspects featured by these fully immersive systems. These technical issues are so impressive that they simply prevail over the cognitive determinants of presence. On the other hand, within non-immersive VEs whose technological infrastructure is less advanced, the user's experience of presence is mainly due not to the system characteristics, but rather to the associated human factors.

The potential set of personality factors which could impact upon sense of presence has been identified after a thorough review of presence literature [15 
12 accompanied by an approach in the psychology of hypnosis. Therefore, we choose to investigate the following factors: empathy, absorption, creative imagination, personality cognitive style and willingness to be transported in the VE. A presentation of each of these factors is briefly outlined below.

- Empathy was identified with a set of constructs associated with the responses of one individual to the experience of another [6]. It involves the ability to engage in the cognitive process of adopting another's psychological point of view, together with the capacity to experience affective reactions to the observed experience of others. In order to develop such capacities and exhibit empathic behaviour, one should be able to assume perceptual, cognitive and affective roles. Empathy was measured with Davis' Interspersonal Reactivity Index 6].

- Absorption construct elaborated by Tellegen [26] is defined as a state of "openness to experiencing, in the sense of readiness to undergo whatever experiential events, sensory or imaginal, that may occur, with a tendency to dwell on, rather than go beyond, the experiences themselves and the objects they represent". Absorption was measured with Tellegen Absorption Scale 27.

- Creative Imagination represents the ability to generate mental representations of objects, persons or events not immediately presented to the senses [24], was also considered to carry an impact upon presence [12]. This trait was measured with Barber and Wilson's Creative Imagination Scale [1].

- Cognitive style. Curry's Onion Model [5] proposes a hierarchical structure of cognitive styles, with the outermost layer referring to the individual's choice of learning environment, with the middle layer referring to information processing style and with the innermost layer consisting of cognitive personality style. Defined as the individual's tendency to assimilate information, cognitive personality style is an enduring and context-independent feature. Personality Cognitive Style was measured with Myers-Briggs Type Indicator (MBTI) [16].

- Willingness to be transported into the virtual world. The role of this factor can be better understood if one looks at it as a prerequisite for willingness to suspend disbelief, aspect often mentioned in relation to sense of presence. It seems to be a necessary condition for experiencing a high level of sense of presence [1425] and ultimately enjoying a mediated experience of any kind (e.g. theatre, literature, television, film, VR).

With respect to the individual differences in experiencing sense of presence, findings indicate that individuals more absorbed $(t(30)=2.10, p<0.05)$, more imaginative $(t(30)=2.41, p<0.05)$, more empathic $(t(30)=2.00, p<0.05)$ or more willing to be transported into the virtual world $(t(30)=2.75, p<$ 0.01 ), experienced a significantly greater level of sense of presence [21. The way in which personality cognitive style shapes the experience of "being there" is indicated by the following results: individuals who are highly introvert $(t(28)=$ 1.68, $p=0.05)$, sensitive $(t(28)=1.47, p=0.07)$ or feeling type $(t(28)=$ $1.46, p=0.07)$ are more prone to experience a higher level of presence [20]. 


\section{Conclusions}

The results presented in this paper indicate the significant impact of some personality factors on spatial performance, navigational patterns and sense of presence experienced by users during their interaction with the system. These findings indicate that "one size fits all" is an obsolete concept when it comes to the design of VEs. Since the ultimate goal of studying individual differences is to enhance system usability, once they have been identified and their impact is significant, efforts should be made to accommodate the individual differences.

In this context, an important aspect to be considered is the purpose of the application. Whether the VE is designed to enable humans to exceed the barrier of our limited senses for allowing access to inaccessible spaces, to improve skills, to cure, or to entertain, greater attention should be paid to different aspects, and accordingly to the set of factors which could trigger remarkable individual differences on these aspects. However, the distinct goal of any VE could be placed on a continuum between two poles: best performance-most pleasure.

When, for instance, the VE is designed for medical applications, its main purpose is to enable surgeons to perform at least as well as they would perform in reality (with or without presence). In this case, particular attention should be given to introvert, sensitive and feeling types who experience a higher level of sense of presence but whose performance might be reduced. For example, a $\mathrm{VE}$ for games should be particularly designed to allow extrovert, intuitive and thinking type individuals not only to perform better in spatial tasks but to experience presence as well.

\section{References}

1. Barber, T.X., Wilson, S.C.: The Barber suggestibility scale and the creative imagination scale: Experimental and clinical applications. The American Journal of Clinical Hypnosis 21(2-3) (1979) 84-108

2. Carroll, J.B.: Human Cognitive Abilities: A Survey of Factor-Analytic Studies. Cambridge University Press, New York (1993)

3. Chen, C.: Individual differences in a spatial-semantic virtual environment. Journal of the American Society for Information Science 51(6) (2000) 529-542

4. Chen, S., Ford, N.: Towards adaptive information systems: Individual differences and hypermedia. Information Research 3(2) (1997)

5. Curry, L.: An organization of learning styles theory and constructs. ERIC Document (1983) 235-185

6. Davis, M.: Empathy: A social psychological approach. Westview Press, Oxford (1994)

7. Dillon, A., Watson, C.: User analysis in HCI: The hystorical lesson from individual differences research. International Journal of Human-Computer Studies 45(6) (1996) 619-637

8. Fernie, K. and Richards, J.D.: Creating and Using Virtual Reality: A Guide for the Arts and Humanities. URL: http://vads.ahds.ac.uk/guides/vr_guide/glossary.html $(10.03 .2003)$ 
9. Ford, N.: Cognitive styles and virtual environments. Journal of American Society for Information Science 51(6) (2000) 543-557

10. Gabbard, J., Hix, D. Taxonomy of Usability Characteristics in Virtual Environments. Virginia Polytechnic Institute, Blackenburg (1997)

11. Goldstein, D., Haldane, D., Mitchell, C.: Sex differences in visual-spatial ability: The role of performance factors. Memory and Cognition 18(5) (1990) 546-550

12. Heeter, C.: Being there: The subjective experience of presence. Presence: Teleoperators and Virtual Environments 1(2) (1992) 262-271

13. Jung, C.G.: Psychological types. In Ress, L., McGuire, W. (eds) The Collected Works of C.G. Jung, Vol. 6 Princeton University Press Princeton (1971)

14. Laurel, B.: Computers as Theatre Addison-Wesley Longman Publishing Co., Inc, Boston (1993)

15. Lombard, M., Ditton, T.: At the heart of it all: The concept of presence. Journal of Computer-Mediated Communication 3(2) (1997)

16. Myers, I.B., McCaulley, M.H.: Manual: A Guide to the Development and Use of the MBTI. Consulting Psychologist Press, Palo Alto, (1998)

17. O'Hare, G.M.P., Sewell, K., Murphy, A., Delahunty, T.: An immersive training experience. In Brusilovsky, P., Stock, O., Strapparava, C. (eds) Lecture Notes in Computer Science Vol. 1892 179-188 Springer-Verlag, Berlin (2000)

18. Revelle, W.: Individual differences. In Kazdin, A. (ed) Encyclopedia of Psychology Oxford University Press (2000)

19. Sas, C. and G.M.P. O'Hare, G.M.P.: The presence equation: An investigation into cognitive factors underlying presence within non-immersive virtual environments. In 4th Annual International Workshop on Presence (2001)

20. Sas, C., O'Hare, G.M.P.: Impact of cognitive style upon sense of presence. In Jacko, J., Stephanidis, C. (eds) Proceedings of the 10th International Conference on Human-Computer Interaction 696-700 (2003)

21. Sas, C., O'Hare, G.M.P.: Presence equation: An investigation into cognitive factor underlying presence Presence: Teleoperators and Virtual Environments 12(5) (2003) in press.

22. Sas, C., O'Hare, G.M.P., Reilly, R.G.: On-line trajectory classification. In Lecture Notes in Computer Science Vol. 2659 1035-1044 Springer-Verlag, Berlin (2003)

23. Schloerb, D.W., Sheridan, T.B.: Experimental investigation of the relationship between subjective telepresence and performance in hand-eye tasks. Telemanipulator and Telepresence Technologies 62-73 (1995)

24. Singer, J.: Imagination. In Kazdin, A. (ed) Encyclopedia of Psychology Vol. 4 227-230 Oxford University Press, Oxford (2000)

25. Slater, M., Usoh, M.: Representations systems, perceptual position, and presence in immersive virtual environments. Presence: Teleoperators and Virtual Environments 2(3) (1993) 221-233

26. Tellegen, A.: Practicing the two disciplines of relaxation and enlightenment: Comment on "Role of the feedback signal in electromyography biofeedback: The relevance of attention" by Qualls and Sheehan. Journal of Experimental Psychology: General 110 (1981) 217-226

27. Tellegen, A.: Brief manual for the differential personality questionnaire. Unpublished manuscript. Department of Psychology, University of Minnesota, Minneapolis (1982)

28. Tromp, J.G.: Methodology of distributed CVE evaluations. Proceedings of UK VR SIG (1997)

29. Waller, D.: Individual differences in spatial learning from computer-simulated environments. Journal of Experimental Psychology: Applied, 8 (2000) 307-321 